\title{
Diet and Skin: A Primer
}

\author{
Sophie A. Greenberg, MD
}

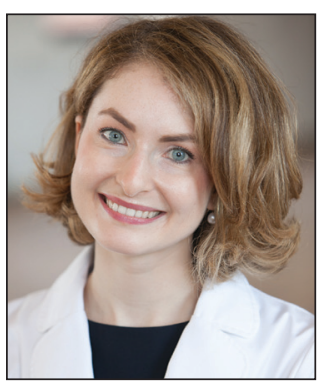

\section{RESIDENT PEARLS}

- There are strong data on the relationship between dietary patterns and skin conditions.

- High glycemic index foods (eg, skim milk, whey protein, sugary beverages, fatty foods) are associated with acne vulgaris.

- Obesity is a risk factor for psoriasis; weight loss interventions such as improved dietary patterns can improve psoriasis.

- Children with atopic dermatitis (AD) are at higher risk for food allergies (both IgE and non-lgE-mediated allergies). A small subset may experience flares in their $A D$ in relation to non-lgE-mediated food allergies.

There are strong data on the relationship between dietary patterns and skin conditions. This article provides an introduction for dermatologists on the role of diet in 3 common skin conditions: acne vulgaris, psoriasis, and atopic dermatitis (AD). High glycemic index foods are associated with acne vulgaris. Psoriasis is associated with obesity and weight loss, and improved dietary patterns may improve cutaneous disease. Children with AD are at higher risk for food allergies, and a small subset may experience flares in relationship to non-IgE-mediated food allergies, though there is a need for further research to clarify which patient subsets require allergen testing and food avoidance.

Cutis. 2020;106:E31-E32.

$\square$ ermatologists frequently learn about skin conditions that are directly linked to diet. For example, we know that nutritional deficiencies can impact the hair, skin, and nails, and that celiac disease manifests with dermatitis herpetiformis of the skin. Patients commonly ask their dermatologists about the impact of diet on their skin. There are many outdated myths, but research on the subject is increasingly demonstrating important associations. Dermatologists must become familiar with the data on this topic so that we can provide informed counseling for our patients. This article reviews the current literature on associations between diet and 3 common cutaneous conditions - acne, psoriasis, and atopic dermatitis [AD]and provides tips on how to best address our patients' questions on this topic.

\section{Acne}

Studies increasingly support an association between a high glycemic diet (foods that lead to a spike in serum glucose) and acne; Bowe et $\mathrm{al}^{1}$ provided an excellent summary of the topic in 2010. This year, a large prospective cohort study of more than 24,000 participants demonstrated an association between adult acne and a diet high in milk, sugary beverages and foods, and fatty foods. ${ }^{2}$ In prospective cohort studies of more than 6000 adolescent girls and 4000 adolescent boys, Adebamowo et al ${ }^{3,4}$ demonstrated a correlation between skim milk consumption and acne. Whey protein supplementation also has been implicated in acne flares. ${ }^{5,6}$ The biological mechanism of the impact of high glycemic index foods and acne is believed to be mainly via activation of the insulinlike growth factor 1 (IGF-1) pathway, which promotes androgen synthesis and increases androgen bioavailability via decreased synthesis of sex hormone binding globulin. ${ }^{1,2}$ Insulinlike growth factor 1 also stimulates its downstream target, mammalian target of rapamycin (mTOR), leading to activation of antiapoptotic and proliferation signaling, ultimately resulting in oxidative stress and inflammation causing acne. ${ }^{2}$ Penso et $\mathrm{al}^{2}$ noted that patients with IGF-1 deficiency (Laron syndrome) never develop acne unless treated with exogenous IGF-1, further supporting its role in acne formation. ${ }^{7}$ There currently is a paucity of randomized controlled trials assessing the impact of diet on acne.

From the Department of Dermatology, Columbia University Medical Center, New York, New York.

The author reports no conflict of interest.

Correspondence: Sophie A. Greenberg, MD, 161 Fort Washington Ave, 12th Floor, New York, NY 10032 (sag2203@cumc.columbia.edu).

doi: $10.12788 /$ cutis. 0143 


\section{Psoriasis}

The literature consistently shows that obesity is a predisposing factor for psoriasis. Additionally, weight gain may cause flares of existing psoriasis. ${ }^{8}$ Promotion of a healthy diet is an important factor in the management of obesity, alongside physical activity and, in some cases, medication and bariatric surgery. ${ }^{9}$ Patients with psoriasis who are overweight have been shown to experience improvement in their psoriasis after weight loss secondary to diet and exercise. ${ }^{8,10}$ The joint American Academy of Dermatology and National Psoriasis Foundation guidelines recommend that dermatologists advise patients to practice a healthy lifestyle including a healthy diet and communicate with a patient's primary care provider so they can be appropriately evaluated and treated for comorbidities including metabolic syndrome, diabetes, and hyperlipidemia. ${ }^{11}$ In the NutriNet-Santé cohort study, investigators found an inverse correlation between psoriasis severity and adherence to a Mediterranean diet, which the authors conclude supports the hypothesis that this may slow the progression of psoriasis. ${ }^{12}$ In a single meta-analysis, it was reported that patients with psoriasis have a 3 -fold increased risk for celiac disease compared to the general population. ${ }^{13}$ It remains unknown if these data are generalizable to the US population. Dermatologists should consider screening patients with psoriasis for celiac disease based on reported symptoms. When suspected, it is necessary to order appropriate serologies and consider referral to gastroenterology prior to recommending a gluten-free diet, as elimination of gluten prior to testing may lead to false-negative results.

\section{Atopic Dermatitis}

Patients and parents/guardians of children with $\mathrm{AD}$ often ask about the impact of diet on the condition. A small minority of patients may experience flares of $\mathrm{AD}$ due to ongoing, non-IgE-mediated allergen exposure. ${ }^{14}$ Diet as a trigger for flares should be suspected in children with persistent, moderate to severe AD. In these patients, allergen avoidance may lead to improvement but not resolution of $\mathrm{AD}$. Allergens ordered from most common to least common are the following: eggs, milk, peanuts/tree nuts, shellfish, soy, and wheat. ${ }^{15}$ Additionally, it is important to note that children with $\mathrm{AD}$ are at higher risk for developing lifethreatening, IgE-mediated food allergies compared to the general population (37\% vs 6.8\%). ${ }^{16,17}$ The LEAP (Learning Early about Peanut Allergy) study led to a paradigm shift in prevention of peanut allergies in high-risk children (ie, those with severe $\mathrm{AD}$ and/or egg allergy), providing data to support the idea that early introduction of allergenic foods such as peanuts may prevent severe allergies. ${ }^{18}$ Further studies are necessary to clarify the population in which allergen testing and recommendations on food avoidance are warranted vs early introduction. ${ }^{19}$

\section{Conclusion}

Early data support the relationship between diet and many common dermatologic conditions, including acne, psoriasis, and AD. Dermatologists should be familiar with the evidence supporting the relationship between diet and various skin conditions to best answer patients' questions and counsel as appropriate. It is important for dermatologists to continue to stay up-to-date on the literature on this subject as new data emerge. Knowledge about the relationship between diet and skin allows dermatologists to not only support our patients' skin health but their overall health as well.

\section{REFERENCES}

1. Bowe WP, Joshi SS, Shalita AR. Diet and acne. J Am Acad Dermatol. 2010;63:124-141.

2. Penso L, Touvier M, Deschasaux M, et al. Association between adult acne and dietary behaviors: findings from the NutriNet-Santé prospective cohort study. JAMA Dermatol. 2020;156:854-862.

3. Adebamowo CA, Spiegelman D, Berkey CS, et al. Milk consumption and acne in teenaged boys. J Am Acad Dermatol. 2008;58:787-793.

4. Adebamowo CA, Spiegelman D, Berkey CS, et al. Milk consumption and acne in adolescent girls. Dermatol Online J. 2006;12:1.

5. Silverberg NB. Whey protein precipitating moderate to severe acne flares in 5 teenaged athletes. Cutis. 2012;90:70-72.

6. Cengiz FP, Cemil BC, Emiroglu N, et al. Acne located on the trunk, whey protein supplementation: is there any association? Health Promot Perspect. 2017;7:106-108.

7. Ben-Amitai D, Laron Z. Effect of insulin-like growth factor-1 deficiency or administration on the occurrence of acne. J Eur Acad Dermatol Venereol. 2011;25:950-954.

Jensen P, Skov L. Psoriasis and obesity [published online February 23, 2017]. Dermatology. 2016;232:633-639.

9. Extreme obesity, and what you can do. American Heart Association website. https://www.heart.org/en/healthy-living/healthy -eating/losing-weight/extreme-obesity-and-what-you-can-do. Updated April 18, 2014. Accessed November 30, 2020.

10. Naldi L, Conti A, Cazzaniga S, et al. Diet and physical exercise in psoriasis: a randomized controlled trial. Br J Dermatol. 2014; 170:634-642.

11. Elmets CA, Leonardi CL, Davis DMR, et al. Joint AAD-NPF guidelines of care for the management and treatment of psoriasis with awareness and attention to comorbidities. J Am Acad Dermatol. 2019;80:1073-1113.

12. Phan C, Touvier M, Kesse-Guyot E, et al. Association between Mediterranean anti-inflammatory dietary profile and severity of psoriasis: results from the NutriNet-Santé cohort. JAMA Dermatol. 2018;154:1017-1024.

13. Ungprasert P, Wijarnpreecha K, Kittanamongkolchai W. Psoriasis and risk of celiac disease: a systematic review and meta-analysis. Indian J Dermatol. 2017;62:41-46.

14. Silverberg NB, Lee-Wong M, Yosipovitch G. Diet and atopic dermatitis. Cutis. 2016;97:227-232.

15. Bieber T, Bussmann C. Atopic dermatitis. In: Bolognia JL, Jorizzo JL, Schaffer JV, eds. Dermatology. 3rd ed. China: Elsevier Saunders; 2012:203-218.

16. Eigenmann PA, Sicherer SH, Borkowski TA, et al. Prevalence of IgE-mediated food allergy among children with atopic dermatitis. Pediatrics. 1998;101:E8.

17. Age-adjusted percentages (with standard errors) of hay fever, respiratory allergies, food allergies, and skin allergies in the past 12 months for children under age 18 years, by selected characteristics: United States, 2016. CDC website. https://ftp.cdc.gov/pub/Health_Statistics/NCHS /NHIS/SHS/2016_SHS_Table_C-2.pdf. Accessed December 8, 2020.

18. Du Toit G, Roberts G, Sayre PH, et al; LEAP study team. Randomized trial of peanut consumption in infants at risk for peanut allergy. $N$ Engl J Med. 2015;372:803-813.

19. Sugita K, Akdis CA. Recent developments and advances in atopic dermatitis and food allergy [published online October 22, 2019]. Allergol Int. 2020;69:204-214. 